\title{
Evaluation of wheat landrace genotypes for salinity tolerance at vegetative stage by using morphological and molecular markers
}

\author{
A. Shahzad ${ }^{1}$, M. Ahmad ${ }^{2}$, M. Iqbal ${ }^{1}$, I. Ahmed ${ }^{1}$ and G.M. Ali ${ }^{1}$ \\ ${ }^{1}$ National Agriculture Research Center, Islamabad, Pakistan \\ ${ }^{2}$ Pir Mehr Ali Shah Arid Agriculture University, Rawalpindi, Pakistan \\ Corresponding author: A. Shahzad \\ E-mail: armghan_shehzad@yahoo.com
}

Genet. Mol. Res. 11 (1): 679-692 (2012)

Received August 1, 2011

Accepted November 17, 2011

Published March 19, 2012

DOI http://dx.doi.org/10.4238/2012.March.19.2

\begin{abstract}
Identification of new sources of salt tolerance is particularly important to develop crop varieties suitable for saline soils. We evaluated 129 Pakistani and 58 exotic wheat landraces/ cultivars grown in Hoagland's hydroponic nutrient solution, under control (tap water equivalent to $10 \mathrm{mM}$ salt) and salt stress (200 $\mathrm{mM} \mathrm{NaCl}$ ) conditions. Forty-four genotypes were also tested under $250 \mathrm{mM} \mathrm{NaCl}$ stress. High heritability and positive correlations suggested that number of tillers per plant, root length, root fresh and dry weights, and shoot fresh and dry weights are associated with salt tolerance and could be used as selection criteria. SSR markers revealed high genetic variation in the wheat genotypes. Twelve SSR markers (cfd 1, cfd 9, cfd 18, cfd 46, cfd 49, cfd 183, wmc 11, wmc 17, wmc 18, wmc 154, wmc 432, and wmc 503) were found to be associated with salt tolerance because they were amplified in tolerant genotypes only. Five markers, cfd 9, cfd 18, cfd 183, wmc 96, and wmc 405, were identified as most suitable to evaluate salt tolerance
\end{abstract}


because they were associated with four or more salt tolerance traits studied. Cultivars Pasban 90, accessions 10790, 10828, 10823, and 4098805 from Pakistan and Sakha-92 from Egypt performed best at both stress levels.

Key words: Salinity tolerance; Hydroponic; Wheat landraces; SSR marker

\section{INTRODUCTION}

Wheat (Triticum aestivum L.) is the major human consumable commodity in most areas of the world including Pakistan. It is a moderately salt-tolerant crop and its yield is substantially reduced as the soil salinity level rises to $100 \mathrm{mM} \mathrm{NaCl}$ (Munns et al., 2006). Over 800 million hectares of land throughout the world (Munns, 2005) and about 6 million hectares in Pakistan (Chatrath et al., 2007) are salt affected. Salinity is a major obstacle to food production because it substantially reduces the average yield of major crops (Ashraf, 2004). Therefore, identification of new sources of salt tolerance may be important for developing saline agriculture. Salinity causes undesirable effects on plant growth, development, physiological, and biochemical activities, which is due to the low osmotic potential of soil solution (osmotic stress), specific ion effects (salt stress), nutritional imbalance, or a combination of these factors (Ashraf, 2004). The ability of plants to tolerate and flourish in saline soils is of great importance in agriculture, because it indicates that the affected plants have genetic potential for salt tolerance, which is a highly desirable trait.

Due to the tremendous heterogeneity of saline soils, screening in the field for salinity tolerance is inefficient and almost impossible so such study should essentially be carried out under controlled conditions. Although all plant growth stages are sensitive to salinity, the seedling stage is considered to be more sensitive in most plant species (Munns, 2002; Cuartero et al., 2006). In addition, this stage is foretelling of plant growth responses to salinity (Cuartero et al., 2006). Therefore, almost all research on salt tolerance in different crop species reported previously (Meneguzzo et al., 2000; Sabir and Ashraf, 2007; El-Hendawy, 2009) had included plant evaluation at this stage. Ali et al. (2007) investigated 98 wheat accessions and found differential growth reductions to increased salinity stress. El-Hendawy et al. (2005) evaluated 13 wheat genotypes and found a significant reduction in number of tillers and dry weight/plant.

Morpho-physiological traits have been used previously to evaluate the genetic diversity for salt tolerance in crop species as mentioned above. However, during the last decade microsatellites or SSR markers have been extensively used for genetic diversity study, genome mapping, varietal identification, etc. Utilization of these markers to investigate the genetic variations and QTL mapping for salt tolerance in different cultivars has been previously reported by some researchers (Liu et al., 2001; Munns, 2002; Ma et al., 2007; Kurup et al., 2009). The potential of these markers to investigate genetic diversity for salinity tolerance in Pakistani wheat landrace genotypes/cultivars is very limited. Pestsova and Roder (2002) used 12 microsatellite markers to study the inheritance in 59 wheat cultivars. Prasad et al. (2000) reported that 12 primer pairs were sufficient to distinguish 48 wheat genotypes.

The present study was initiated to evaluate the performance of wheat landrace genotypes under hydroponic salinity at control (tap water), 200 and $250 \mathrm{mM} \mathrm{NaCl}$ stress and by SSR markers so that these may be utilized to develop salt-tolerant cultivars either by conventional or molecular approaches. 


\section{MATERIAL AND METHODS}

Seeds of 187 wheat landraces/cultivars (Table 2) including seven check cultivars/lines, namely, Shorawaki, Pasban 90, SARC III, SARC IV, SARC VII, LU 26S, and 22284 were acquired from the gene bank of the Plant Genetic Resources Program (NARC), University of Agriculture Faisalabad and Ayyub Agricultural Research Institute, Faisalabad. The landraces/cultivars included 129 accessions from Pakistan, 24 from Iran, 14 from Syria, six from Egypt, seven from Italy, two from the USA, one from Brazil, and four from Mexico. In the first phase of the experiment all the accessions were tested under two treatments, viz., control (tap water equivalent to $10 \mathrm{mM}$ salt) and $200 \mathrm{mM}$ salt $(\mathrm{NaCl})$ stress. Seeds were germinated in sand-filled steel trays. Ten-day-old uniformly germinated seedlings were wrapped in foam and transplanted to holes made in thin styrofoam sheets. Plants floated over the half strength Hoagland's nutrient solution (Hoagland and Arnon, 1950) in a 200-L steel tank lined internally with polyethylene sheets. The $\mathrm{pH}$ of the culture solution was maintained daily between 5.5-6.0 with $1 \mathrm{~N} \mathrm{HCl}$ or $\mathrm{KOH}$. Culture solution was aerated for $24 \mathrm{~h}$ with air pumps throughout the growing period. Each treatment was replicated thrice following a completely randomized design. Salinity was developed in increments of $50 \mathrm{mM}$ daily until it reached $200 \mathrm{mM}$ with the addition of $\mathrm{NaCl}$. Plants were harvested 30 days after salt treatment. Data were recorded on shoot length, root length, No. of tillers/plant, shoot fresh and dry weights, and root fresh and dry weights. Salt tolerance trait index (STTI) at the seedling stage was calculated following Ali et al. (2007).

$$
\text { STTI }=\frac{\text { Value of trait under stress condition }}{\text { Value of trait under controlled condition }} \times 100
$$

Salt tolerance index (STI) was calculated to be $39 \%$ as the mean of STTIs. The data were subjected to analysis of variance, heritability estimation and genotypic and phenotypic correlation by using the SAS statistical software (SAS Institute, 2003). The means obtained were separated by standard error of differences of means. Based on STI, 187 genotypes were divided into four categories. Difference between the range of STI was divided into four equal groups (Table 1), namely, salt tolerant (STI $=39 \%$ and above), moderately salt tolerant $(\mathrm{STI}=30-39 \%)$, moderately salt susceptible (STI $=21-30 \%$ ), and salt susceptible (STI $=21 \%$ and below).

In the second phase of the experiment, 89 genotypes ( 47 salt tolerant and 42 sensitive) selected from the 187 genotypes on the basis of their relative performance were evaluated at 250 $\mathrm{mM}$ salt stress. Only 44 genotypes survived at $250 \mathrm{mM} \mathrm{NaCl}$ stress. The same procedure for data collection and analysis was followed as mentioned for the first phase of the experiment. The STI data of 44 surviving genotypes were used to rank these genotypes. A total of 26 (18 salt tolerant and 8 sensitive) genotypes were selected for SSR studies (Table 2). These genotypes were selected from the evaluation at early germination stage under 200,250 and $300 \mathrm{mM} \mathrm{NaCl}$, evaluation at vegetative stage under 200 and $250 \mathrm{mM} \mathrm{NaCl}$ stress and field evaluation for two years at two salt-affected locations. One salt-susceptible genotype, 'PBW 343', was acquired from Dr. Mujeeb Kazi, Wheat Wide Crosses Program National Agricultural Research Center, Islamabad, Pakistan (Figure 1). DNA isolation, purification, and quantification were carried out following Shah et al. (2009). PCRs were carried out according to the method described by Shah et al. (2009), with some modification. A total of 240 SSR primer pairs were optimized with two bulked DNA of salt-tolerant and -susceptible genotypes. Only 45 SSR primers amplified polymorphic amplicons (Table 6). PCR products were separated on $1.5 \%$ agarose gel. PCR products were visualized under 
UV pro-transiluminator, photographed and scanned. For statistical analysis of SSR data the scoreable bands were considered as a single locus/allele. The loci were scored as present (1) or absent (0). Polymorphic information content (PIC) value of the SSR marker was calculated following the method developed by Anderson et al. (1993). Pairwise comparisons of the cultivars based on the proportion of amplification products (alleles) were used to measure the genetic similarity by Dice coefficients. The Dice coefficients were computed by using the Simqual subprogram in similarity routine of NTSYS-pc, version 2.2 (Rohlf, 2005). The resultant similarity matrix data were used to construct a dendrogram by using sequential agglomerative hierarchical nesting (SAHN) based on unweighted pair-group method with an arithmetic average (UPGMA) to infer genetic relationships and phylogeny among genotypes. Bivariate SSR data and STTI data recorded under $200 \mathrm{mM}$ stress were subjected to analysis of variance by using MINITAB 13 .

\section{RESULTS}

Plant growth was determined by number of tillers/plant, root and shoot lengths and fresh and dry weights. At $200 \mathrm{mM} \mathrm{NaCl}$ stress, STTI of shoot length ranged from 17.4 to $61.5 \%$. Under stress conditions, roots play an important role in plant survival. STTI for root length ranged from 23.4 to $74.3 \%$. This wide range indicated that genotypes had broad genetic base for root length. Overall, salt-tolerant genotypes showed greater root growth than susceptible genotypes. STTI of number of tillers/plant ranged from 9.5 to $80.0 \%$. Fresh shoot and root weight were also adversely affected by $200 \mathrm{mM} \mathrm{NaCl}$ stress. STTI of fresh shoot weight ranged from 1.3 to $23.1 \%$ and that of fresh root weight from 3.3 to $49.6 \%$ (data not shown). STTI of dry shoot weight ranged from 0.9 to $35.3 \%$ and that of dry root weight ranged from 4.0 to $61.4 \%$. From the 187 genotypes only 5 accessions fell into the tolerant category, one from Iran and four from Pakistan. Fifty-nine accessions were moderately salt tolerant, 80 accessions were moderately salt susceptible and 34 accessions were salt susceptible (Table 1).

At $250 \mathrm{mM} \mathrm{NaCl}$ stress STTI for shoot length ranged from 27.5 to $77.5 \%$. STTI for root length ranged from 22.6 to $52.1 \%$ (Table 2). Number of tillers/plant reduced significantly under $250 \mathrm{mM} \mathrm{NaCl}$ stress as it ranged from 12.0 to $78.6 \%$. STTI for shoot fresh weight ranged from 3.9 to $35.2 \%$. Fresh root weight was also adversely affected by $250 \mathrm{mM} \mathrm{NaCl}$ stress but the effect was less as compared to fresh shoot weight (Table 2). STTI for fresh root weight ranged from 9.2 to 48.0\%. Check cultivar Pasban 90 acquired 35.7\% STTI for fresh root weight. STTI for dry shoot weight ranged from 5.1 to $43.1 \%$. STTI for dry root weight ranged from 12.2 to $70.6 \%$ (Table 2).

Heritability estimates under stress conditions were found to be lower than under controlled conditions during both phases of the experiment (Table 3). This indicated that heritability is not constant and varies with changes in environment. Heritability estimates under 200 $\mathrm{mM} \mathrm{NaCl}$ were above $80 \%$ for shoot fresh and dry weights and root fresh weight. Under 250 $\mathrm{mM} \mathrm{NaCl}$ stress heritability estimates were above $60 \%$ for shoot length, tillers/plant, shoot fresh and dry weights and root fresh weight. Shoot length was positively and significantly ( $\mathrm{P}$ $<0.05)$ correlated with root length, and shoot fresh and dry weight at control and $200 \mathrm{mM}$ $\mathrm{NaCl}$ stress. However, shoot length and number of tillers were negatively correlated at control and both stress levels. Root length showed significant $(\mathrm{P}<0.05)$ positive phenotypic and genotypic correlation under control conditions with No. of tillers/plant, shoot fresh and dry weights, and root fresh and dry weights. Phenotypic correlation was also significant $(\mathrm{P}<0.05)$ with tillers/plant, shoot and root dry weight under $200 \mathrm{mM} \mathrm{NaCl}$ stress (Table 4). 


\begin{tabular}{|c|c|c|c|}
\hline Salt tolerance category & Range of STI & $\begin{array}{l}\text { No. of } \\
\text { accessions }\end{array}$ & Accessions (Country of collection) \\
\hline Tolerant & $39 \%$ and above & 5 & Sakha 92 (Egypt), 10793 (Pak), 10790 (Pak), 10821 (Pak) and 11526 (Pak) \\
\hline Moderately tolerant & $39-30 \%$ & 59 & $\begin{array}{l}\text { Local white (Pak), Parula (Mexico), Karaj-II (Iran), } 10828 \text { (Pak), } 10806 \text { (Pak), } \\
10824 \text { (Pak), } 10810 \text { (Pak), } 4098775 \text { (Pak), } 10841 \text { (Pak), } 10812 \text { (Pak), } \\
\text { Pasban } 90 \text { (Pak), Omid (Iran), } 4098785 \text { (Pak), Shorawaki (Pak), } \\
\text { Roushan (Iran), } 10823 \text { (Pak), } 10805 \text { (Pak), } 10833 \text { (Pak), } 11242 \text { (Pak), } \\
10789 \text { (Pak), } 10839 \text { (Pak), } 10831 \text { (Pak), } 11214 \text { (Pak), } 10771 \text { (Pak), } \\
10832 \text { (Pak), } 11383 \text { (Pak), } 10826 \text { (Pak), } 10840 \text { (Pak), } 11195 \text { (Pak), } 10807 \text { (Pak), } \\
11290 \text { (Pak), Pavon (Mexico), Maroon (Iran) } 10795 \text { (Pak), } 11373 \text { (Italy), } \\
\text { Sakha } 61 \text { (Egypt), } 10770 \text { (Pak), } 11454 \text { (Pak), } 11221 \text { (Pak), } 11466 \text { (Pak), } \\
10850 \text { (Pak), } 10851 \text { (Pak), } 10829 \text { (Pak), 11406 (Pak) Chakwal } 86 \text { (Pak), } \\
10809 \text { (Pak), } 11244 \text { (Pak), India (Iran), } 11133 \text { (Pak), 10859 (Pak), } \\
11193 \text { (Pak), } 10835 \text { (Pak), } 10843 \text { (Pak), } 10792 \text { (Pak), Giza-163 (Egyp), } \\
11186 \text { (Pak), } 10849 \text { (Pak), } 11901 \text { (Italy) and LU26S (Pak), }\end{array}$ \\
\hline Moderately susceptible & $30-21 \%$ & 80 & $\begin{array}{l}11401 \text { (Pak), Azadi (Iran), } 10819 \text { (Pak), Giza-155 (Egypt), } 10861 \text { (Pak), } \\
11478 \text { (Italy), } 10772 \text { (Pak), } 10815 \text { (Pak), Golestan (Iran), } 10791 \text { (Pak), } \\
11299 \text { (Pak), } 10775 \text { (Pak), } 10830 \text { (Pak), } 10783 \text { (Pak), } 11453 \text { (Syria), } \\
4098795 \text { (Pak), } 11372 \text { (Italy), } 11289 \text { (Pak), } 4098825 \text { (Pak), } 10862 \text { (Pak), } \\
10786 \text { (Pak), Yecora-70 (Pak), } 11465 \text { (Syria), } 11369 \text { (Italy), Barrei (Canada), } \\
11460 \text { (Syria), } 10811 \text { (Pak), } 11522 \text { (Pak), } 11240 \text { (Pak), 10813 (Pak), } \\
4098815 \text { (Pak), } 11171 \text { (Pak), } 10853 \text { (Pak), } 11461 \text { (Syria), } 11387 \text { (Pak), } \\
\text { Darab (Iran), Bezostaya (Iran), Kohistan-97 (Pak), 10801 (Pak), 11416 (Pak), } \\
11374 \text { (Italy), 11555 (Pak), 10784 (Pak), Arvand (Iran), SARC-V (Pak), } \\
\text { SARC-III (Pak), Falat (Iran), } 11379 \text { (Pak), } 11419 \text { (Pak), 11370 (Italy), } \\
\text { Adl (Iran), 11380 (Pak), Sardari (Iran), 11407 (Pak), Moghan-I (Iran), } \\
\text { SARC-I (Pak), SARC VII (Pak), Rasool (Iran), } 10777 \text { (Pak), Tabasi (Iran), } \\
11287 \text { (Pak), } 10798 \text { (Pak), } 4098835 \text { (Pak), Alborz (Iran), Hirmand (Iran), } \\
11467 \text { (Syria), } 11248 \text { (Pak), Era (USA),10803 (Pak), 11302 (Pak), } \\
\text { Sakha-69 (Egypt), } 11417 \text { (Pak), Sabalan (Iran), } 11403 \text { (Pak), } 11384 \text { (Pak), } \\
11546 \text { (Pak), } 11386 \text { (Pak), 11415 (Pak), Bule silver (Pak), and } 11399 \text { (Pak) }\end{array}$ \\
\hline Susceptible & $21 \%$ and below & 34 & $\begin{array}{l}11459 \text { (Pak), } 11408 \text { (Pak), } 11414 \text { (Pak), Frontana (Brazil), Chakwal } 97 \text { (Pak), } \\
11388 \text { (Pak), } 11385 \text { (Pak), } 11462 \text { (Pak), } 11456 \text { (Pak), } 10854 \text { (Pak), } \\
\text { Tobari } 66 \text { (Mexico), } 11464 \text { (Pak), Bayat (Iran), Chenab (Iran), LU-26 (Pak), } \\
10767 \text { (Pak), } 4098855 \text { (Pak), } 11402 \text { (Pak), } 11418 \text { (Pak), } 11409 \text { (Pak), } \\
11378 \text { (Pak), SARC-IV (Pak), Cutler (Mexico), Chods (Iran), } 4098865 \text { (Pak), } \\
11463 \text { (Syria), } 4098875 \text { (Pak), } 11400 \text { (Pak), } 11382 \text { (Pak), } 11371 \text { (Italy), } \\
11381 \text { (Pak), } 11457 \text { (Syria), } 11458 \text { (Syria), and } 22284 \text { (Pak) }\end{array}$ \\
\hline
\end{tabular}

In the second phase of the experiment, root length exhibited positive correlation at the genotypic and phenotypic level with all the traits studied under control and $250 \mathrm{mM} \mathrm{NaCl}$ stress (Table 5). Number of tillers/plant exhibited positive correlation with all the traits under control conditions, 200 and $250 \mathrm{mM} \mathrm{NaCl}$ stress except shoot length. However, genotypic correlation was significant $(\mathrm{P}<0.05)$ only with root fresh and dry weights under control conditions. Shoot fresh weight significantly $(\mathrm{P}<0.05)$ and positively correlated with shoot dry weight and root fresh and dry weights under control and $200 \mathrm{mM} \mathrm{NaCl}$ at both genotypic and phenotypic level. However, in the second phase of the experiment shoot fresh weight, although exhibiting positive genotypic and phenotypic correlation with all traits, was significantly $(\mathrm{P}<$ 0.05 ) correlated with shoot dry weight at control and $250 \mathrm{mM} \mathrm{NaCl}$ stress. Root fresh weight showed significantly $(\mathrm{P}<0.05)$ positive correlation at both genotypic and phenotypic levels with shoot dry weight and root dry weight under control and $200 \mathrm{mM} \mathrm{NaCl}$ stress. However, at $250 \mathrm{mM} \mathrm{NaCl}$ stress phenotypic correlation with root dry weight was significant $(\mathrm{P}<0.05)$. Shoot dry weight positively correlated with all the traits studied but significantly $(\mathrm{P}<0.05)$ correlated with root dry weight under control and $200 \mathrm{mM} \mathrm{NaCl}$ stress. 


\begin{tabular}{|c|c|c|c|c|c|c|c|c|c|c|c|}
\hline Sr. No. & Accession No. & $\begin{array}{l}\text { Area of } \\
\text { collection }\end{array}$ & $\begin{array}{l}\text { Shoot } \\
\text { length }\end{array}$ & $\begin{array}{c}\text { Root } \\
\text { length }\end{array}$ & $\begin{array}{c}\text { Tillers/ } \\
\text { plant }\end{array}$ & $\begin{array}{c}\text { Fresh shoot } \\
\text { weight }\end{array}$ & $\begin{array}{c}\text { Fresh root } \\
\text { weight }\end{array}$ & $\begin{array}{c}\text { Dry shoot } \\
\text { weight }\end{array}$ & $\begin{array}{c}\text { Dry root } \\
\text { weight }\end{array}$ & STI & Rank \\
\hline 1 & 10767 & Faisalabad & 50.3 & 52.1 & 50.0 & 11.0 & 27.4 & 9.7 & 20.5 & 31.6 & 16 \\
\hline 2 & 10775 & Faisalabad & 41.6 & 28.8 & 43.8 & 8.9 & 28.0 & 12.8 & 36.8 & 28.7 & 21 \\
\hline 3 & 10783 & Faisalabad & 49.1 & 35.9 & 29.0 & 7.9 & 27.2 & 14.3 & 17.8 & 25.9 & 26 \\
\hline 4 & 10786 & Faisalabad & 52.1 & 22.6 & 50.0 & 11.7 & 42.9 & 22.0 & 42.2 & 34.8 & 10 \\
\hline 5 & 10790 & Faisalabad & 41.4 & 37.0 & 65.7 & 8.9 & 30.1 & 19.2 & 28.1 & 32.9 & 13 \\
\hline 6 & 10793 & Faisalabad & 33.9 & 25.2 & 43.6 & 6.5 & 15.4 & 11.0 & 21.6 & 22.5 & 36 \\
\hline 7 & 10795 & Faisalabad & 36.4 & 26.8 & 32.4 & 7.6 & 21.4 & 14.9 & 26.9 & 23.8 & 33 \\
\hline 8 & 10806 & Faisalabad & 42.4 & 36.2 & 13.2 & 3.9 & 9.7 & 6.9 & 20.6 & 19.0 & 42 \\
\hline 9 & 10807 & Faisalabad & 44.0 & 31.1 & 55.0 & 16.8 & 19.8 & 18.1 & 29.4 & 30.6 & 18 \\
\hline 10 & 10811 & Faisalabad & 48.2 & 39.8 & 29.0 & 11.1 & 22.9 & 17.4 & 26.5 & 27.8 & 23 \\
\hline 11 & 10812 & Faisalabad & 48.6 & 34.1 & 19.4 & 7.5 & 11.5 & 7.6 & 16.7 & 20.8 & 39 \\
\hline 12 & 10813 & Faisalabad & 34.1 & 37.6 & 41.4 & 7.3 & 19.8 & 13.5 & 24.1 & 25.4 & 27 \\
\hline 13 & 10823 & Faisalabad & 42.1 & 28.2 & 50.0 & 12.9 & 33.2 & 21.4 & 49.5 & 33.9 & 11 \\
\hline 14 & 10826 & Faisalabad & 35.9 & 24.3 & 25.0 & 7.1 & 20.4 & 9.1 & 23.9 & 20.8 & 38 \\
\hline 15 & 10828 & Faisalabad & 44.7 & 42.6 & 48.3 & 15.4 & 37.9 & 12.9 & 34.3 & 33.7 & 12 \\
\hline 16 & 10831 & Faisalabad & 44.0 & 36.2 & 12.0 & 6.2 & 9.2 & 6.1 & 13.0 & 18.1 & 44 \\
\hline 17 & 10849 & Faisalabad & 39.5 & 39.3 & 38.5 & 12.6 & 31.6 & 18.1 & 45.1 & 32.1 & 14 \\
\hline 18 & 10851 & Faisalabad & 47.3 & 22.7 & 21.3 & 4.2 & 13.8 & 5.1 & 14.6 & 18.4 & 43 \\
\hline 19 & 10859 & Faisalabad & 41.0 & 29.7 & 23.5 & 6.1 & 14.8 & 10.0 & 12.2 & 19.6 & 41 \\
\hline 20 & 11186 & Kharan & 50.8 & 41.6 & 38.2 & 13.6 & 24.3 & 19.5 & 31.1 & 31.3 & 17 \\
\hline 21 & 11214 & Loralai & 77.5 & 37.6 & 27.3 & 35.2 & 26.1 & 43.1 & 28.2 & 39.3 & 5 \\
\hline 22 & 11299 & Chagai & 51.3 & 34.1 & 78.6 & 18.6 & 46.3 & 26.8 & 70.6 & 46.6 & 1 \\
\hline 23 & 11335 & Chagai & 46.3 & 39.6 & 51.7 & 27.1 & 48.0 & 30.5 & 38.7 & 40.3 & 2 \\
\hline 24 & 11369 & Italy & 52.5 & 29.0 & 27.8 & 6.7 & 17.9 & 12.1 & 25.2 & 24.4 & 29 \\
\hline 25 & 11370 & Italy & 47.3 & 41.1 & 47.1 & 24.1 & 46.3 & 22.3 & 50.5 & 39.8 & 3 \\
\hline 26 & 11371 & Italy & 35.0 & 31.1 & 38.1 & 11.1 & 24.1 & 8.9 & 43.5 & 27.4 & 24 \\
\hline 27 & 11373 & Italy & 46.5 & 34.5 & 46.7 & 21.2 & 37.8 & 26.1 & 35.6 & 35.5 & 9 \\
\hline 28 & 11403 & Larkana & 40.4 & 29.1 & 30.0 & 6.0 & 10.9 & 5.8 & 21.1 & 20.5 & 40 \\
\hline 29 & 11417 & Bhakkar & 44.6 & 34.9 & 29.2 & 11.0 & 15.0 & 11.0 & 22.5 & 24.0 & 31 \\
\hline 30 & 11454 & Syria & 42.0 & 46.4 & 36.0 & 8.7 & 20.9 & 11.5 & 33.5 & 28.4 & 22 \\
\hline 31 & 11464 & Syria & 42.5 & 41.3 & 26.7 & 13.3 & 17.3 & 15.6 & 32.7 & 27.1 & 25 \\
\hline 32 & 11466 & Egypt & 44.9 & 30.0 & 23.8 & 7.3 & 17.2 & 8.4 & 18.5 & 21.4 & 37 \\
\hline 33 & 4098775 & Faisalabad & 48.2 & 33.7 & 38.5 & 12.4 & 31.3 & 18.0 & 32.1 & 30.6 & 19 \\
\hline 34 & 4098805 & Faisalabad & 52.1 & 45.7 & 46.7 & 15.6 & 33.5 & 18.5 & 46.2 & 36.9 & 8 \\
\hline 35 & Bayat & IRAN & 47.8 & 37.5 & 50.0 & 10.8 & 31.7 & 11.4 & 32.8 & 31.7 & 15 \\
\hline 36 & Karaj-II & IRAN & 47.0 & 30.9 & 21.7 & 10.1 & 18.3 & 13.3 & 25.8 & 23.9 & 32 \\
\hline 37 & Maroon & IRAN & 48.8 & 32.0 & 45.5 & 21.3 & 42.9 & 24.2 & 52.8 & 38.2 & 6 \\
\hline 38 & Omid & IRAN & 27.5 & 35.6 & 30.0 & 10.6 & 31.5 & 26.7 & 40.7 & 28.9 & 20 \\
\hline 39 & Pasban 90 & Pakistan & 56.3 & 43.5 & 28.0 & 26.6 & 35.7 & 33.4 & 53.1 & 39.5 & 4 \\
\hline 40 & Roushan & IRAN & 37.8 & 27.5 & 27.6 & 8.6 & 20.8 & 9.9 & 32.1 & 23.5 & 35 \\
\hline 41 & 22284 & Faisalabad & 45.1 & 32.5 & 18.8 & 8.6 & 14.9 & 11.6 & 34.2 & 23.7 & 34 \\
\hline 42 & Sakha 92 & Egypt & 55.8 & 35.6 & 44.4 & 15.6 & 44.1 & 19.0 & 46.5 & 37.3 & 7 \\
\hline 43 & SARC IV & Pakistan & 54.8 & 34.7 & 16.1 & 12.1 & 20.6 & 9.9 & 27.6 & 25.1 & 28 \\
\hline 44 & Shorawaki & Pakistan & 35.2 & 45.4 & 22.7 & 8.9 & 23.1 & 9.0 & 24.1 & 24.1 & 30 \\
\hline $\mathrm{SE}_{\text {diff }}$ & & & 1.22 & 1.00 & 2.13 & 0.99 & 1.62 & 1.22 & 1.87 & 1.06 & \\
\hline
\end{tabular}

$\mathrm{SE}_{\text {diff }}=$ standard error of the difference between STTI/STI.

Table 3. Heritability $\left(\mathrm{h}^{2}\right)$ narrow $(\mathrm{N})$ sense $\pm \mathrm{SE}$ of seven agronomic traits under 200 and $250 \mathrm{mM} \mathrm{NaCl}$ stress.

\begin{tabular}{|c|c|c|c|c|c|c|c|}
\hline & Shoot length & Root length & Tillers/plant & $\begin{array}{c}\text { Shoot fresh } \\
\text { weight }\end{array}$ & $\begin{array}{c}\text { Root fresh } \\
\text { weight }\end{array}$ & $\begin{array}{l}\text { Shoot dry } \\
\text { weight }\end{array}$ & $\begin{array}{l}\text { Root dry } \\
\text { weight }\end{array}$ \\
\hline $\mathrm{h}^{2} \mathrm{~N}$ (control) (first phase) & $0.81 \pm 0.02$ & $0.67 \pm 0.03$ & $0.91 \pm 0.01$ & $0.97 \pm 0.00$ & $0.98 \pm 0.00$ & $0.75 \pm 0.02$ & $0.87 \pm 0.02$ \\
\hline $\mathrm{h}^{2} \mathrm{~N}(200 \mathrm{mM} \mathrm{NaCl})$ (first phase) & $0.56 \pm 0.04$ & $0.40 \pm 0.04$ & $0.40 \pm 0.04$ & $0.83 \pm 0.02$ & $0.85 \pm 0.02$ & $0.81 \pm 0.02$ & $0.51 \pm 0.40$ \\
\hline $\mathrm{h}^{2} \mathrm{~N}$ (control) (second phase) & $0.85 \pm 0.04$ & $0.72 \pm 0.06$ & $0.92 \pm 0.02$ & $0.94 \pm 0.02$ & $0.85 \pm 0.03$ & $0.94 \pm 0.02$ & $0.79 \pm 0.05$ \\
\hline $\mathrm{h}^{2} \mathrm{~N}(250 \mathrm{mM} \mathrm{NaCl})$ (second phase) & $0.60 \pm 0.08$ & $0.21 \pm 0.09$ & $0.78 \pm 0.04$ & $0.67 \pm 0.07$ & $0.67 \pm 0.07$ & $0.73 \pm 0.06$ & $0.59 \pm 0.07$ \\
\hline
\end{tabular}


Table 4. Phenotypic (p) and genotypic (g) correlation coefficient among 7 agronomic traits in wheat at 200 $\mathrm{mM}$ salt stress.

\begin{tabular}{|c|c|c|c|c|c|c|c|c|c|c|c|c|}
\hline & \multicolumn{6}{|c|}{ Control } & \multicolumn{6}{|c|}{$200 \mathrm{mM} \mathrm{NaCl}$ stress } \\
\hline & $\begin{array}{l}\text { Root } \\
\text { length }\end{array}$ & $\begin{array}{l}\text { No. of tillers/ } \\
\text { plant }\end{array}$ & $\begin{array}{c}\text { Shoot fresh } \\
\text { weight }\end{array}$ & $\begin{array}{c}\text { Root fresh } \\
\text { weight }\end{array}$ & $\begin{array}{c}\text { Shoot dry } \\
\text { weight }\end{array}$ & $\begin{array}{c}\text { Root dry } \\
\text { weight }\end{array}$ & $\begin{array}{l}\text { Root } \\
\text { length }\end{array}$ & $\begin{array}{l}\text { No. of tillers/ } \\
\text { plant }\end{array}$ & $\begin{array}{c}\text { Shoot fresh } \\
\text { weight }\end{array}$ & $\begin{array}{c}\text { Root fresh } \\
\text { weight }\end{array}$ & $\begin{array}{c}\text { Shoot dry } \\
\text { weight }\end{array}$ & $\begin{array}{c}\text { Root dry } \\
\text { weight }\end{array}$ \\
\hline \multirow[t]{2}{*}{ Shoot length } & p $0.22 *$ & $\mathrm{p}-0.07$ & p $0.37^{*}$ & p 0.17 & p $0.31^{*}$ & p 0.11 & p $0.29 *$ & $\mathrm{p}-0.04$ & p $0.12 *$ & p 0.19 & p $0.28^{*}$ & p $0.18^{*}$ \\
\hline & g 0.23 & g - -0.09 & g 0.40 & g 0.19 & g 0.35 & g 0.11 & g 0.31 & $\mathrm{~g} 0.09^{*}$ & g 0.04 & g - -0.08 & g 0.30 & g 0.14 \\
\hline \multirow[t]{2}{*}{ Root length } & & p $0.36^{*}$ & p 0.44* & p $0.47^{*}$ & p $0.51^{*}$ & p $0.48^{*}$ & & p $0.13^{*}$ & p 0.06 & $\mathrm{p}-0.08$ & p $0.08^{*}$ & p $0.04 *$ \\
\hline & & g 0.43 & $\operatorname{g~} 0.54 *$ & g $0.58^{*}$ & g $0.64^{*}$ & $\mathrm{~g} 0.58^{*}$ & & g 0.20 & $\mathrm{~g}-0.18$ & $\mathrm{~g}-0.19$ & g 0.06 & g - 0.07 \\
\hline \multirow[t]{2}{*}{ No. of tillers/plant } & & & p $0.66^{*}$ & p $0.73^{*}$ & p $0.62^{*}$ & p $0.66^{*}$ & & & $\mathrm{p} 0.37^{*}$ & $\mathrm{p} 0.33^{*}$ & p $0.44^{*}$ & p $0.27^{*}$ \\
\hline & & & g 0.70 & g $0.77^{*}$ & g 0.72 & g 0.72* & & & g 0.53 & g 0.42 & g 0.60 & g 0.38 \\
\hline \multirow[t]{2}{*}{ Shoot fresh weight } & & & & p $0.86^{*}$ & p $0.81^{*}$ & p $0.76^{*}$ & & & & p $0.72 *$ & p $0.67^{*}$ & p $0.49^{*}$ \\
\hline & & & & g 0.88* & g 0.93* & g $0.82 *$ & & & & g 0.83* & g $0.79^{*}$ & g $0.75^{*}$ \\
\hline \multirow[t]{2}{*}{ Root fresh weight } & & & & & p $0.80^{*}$ & p $0.90^{* *}$ & & & & & p $0.62^{*}$ & p $0.58^{*}$ \\
\hline & & & & & g $0.91^{*}$ & g $0.95^{* *}$ & & & & & g 0.68* & g $0.78^{*}$ \\
\hline \multirow[t]{2}{*}{ Shoot dry weight } & & & & & & p $0.77^{*}$ & & & & & & p $0.52 \%$ \\
\hline & & & & & & g $0.89^{*}$ & & & & & & g $0.70^{*}$ \\
\hline
\end{tabular}

*Significant at $5 \%$ probability level. **Significant at $1 \%$ probability level.

Table 5. Phenotypic (p) and genotypic (g) correlation coefficient among 7 agronomic traits in wheat at $250 \mathrm{mM}$ salt stress.

\begin{tabular}{|c|c|c|c|c|c|c|c|c|c|c|c|c|}
\hline & \multicolumn{6}{|c|}{ Control } & \multicolumn{6}{|c|}{$250 \mathrm{mM} \mathrm{NaCl}$ stress } \\
\hline & $\begin{array}{l}\text { Root } \\
\text { length }\end{array}$ & $\begin{array}{l}\text { No. of tillers/ } \\
\text { plant }\end{array}$ & $\begin{array}{l}\text { Shoot fresh } \\
\text { weight }\end{array}$ & $\begin{array}{c}\text { Root fresh } \\
\text { weight }\end{array}$ & $\begin{array}{c}\text { Shoot dry } \\
\text { weight }\end{array}$ & $\begin{array}{c}\text { Root dry } \\
\text { weight }\end{array}$ & $\begin{array}{l}\text { Root } \\
\text { length }\end{array}$ & $\begin{array}{l}\text { No. of tillers/ } \\
\text { plant }\end{array}$ & $\begin{array}{c}\text { Shoot fresh } \\
\text { weight }\end{array}$ & $\begin{array}{c}\text { Root fresh } \\
\text { weight }\end{array}$ & $\begin{array}{l}\text { Shoot dry } \\
\text { weight }\end{array}$ & $\begin{array}{c}\text { Root dry } \\
\text { weight }\end{array}$ \\
\hline \multirow[t]{2}{*}{ Shoot length } & p 0.26 & $\mathrm{p}-0.003$ & p 0.48 & p 0.16 & p 0.41 & p 0.20 & p 0.16 & $\mathrm{p}-0.17$ & p 0.44 & p 0.40 & p 0.40 & p 0.24 \\
\hline & g 0.28 & $\mathrm{~g}-0.03$ & g 0.51 & g 0.13 & g 0.44 & g 0.21 & $\mathrm{~g}-0.14$ & $\mathrm{~g}-0.32$ & g 0.35 & g 0.32 & g 0.38 & g 0.17 \\
\hline \multirow[t]{2}{*}{ Root length } & & p 0.44 & p 0.36 & p 0.25 & p 0.24 & p 0.30 & & p 0.27 & p 0.38 & $\mathrm{p} 0.33$ & p $0.35^{* *}$ & p 0.32 \\
\hline & & g 0.52 & g 0.41 & g 0.27 & g 0.27 & g 0.31 & & g 0.44 & g 0.55 & g 0.53 & g $0.55^{* *}$ & g 0.45 \\
\hline \multirow[t]{2}{*}{ No. of tillers/plant } & & & p 0.62 & p 0.70 & p 0.45 & p 0.68 & & & p 0.45 & p 0.61 & p 0.51 & p 0.52 \\
\hline & & & g 0.64 & g 0.74 & g 0.46 & g 0.74 & & & g 0.45 & g 0.63 & g 0.53 & g 0.55 \\
\hline \multirow[t]{2}{*}{ Shoot fresh weight } & & & & p 0.70 & p $0.87^{*}$ & p 0.79 & & & & p $0.82 *$ & p $0.81^{*}$ & p 0.78 \\
\hline & & & & g 0.73 & g 0.89* & g 0.86 & & & & g 0.86 & g $0.83^{*}$ & g 0.86 \\
\hline \multirow[t]{2}{*}{ Root fresh weight } & & & & & p 0.76 & p $0.85^{*}$ & & & & & p $0.84^{*}$ & p 0.73* \\
\hline & & & & & g 0.81 & g $0.95^{*}$ & & & & & g $0.89^{*}$ & g 0.79 \\
\hline \multirow[t]{2}{*}{ Shoot dry weight } & & & & & & $\mathrm{p} 0.75$ & & & & & & p 0.71 \\
\hline & & & & & & g 0.81 & & & & & & g 0.74 \\
\hline
\end{tabular}

*Significant at $5 \%$ probability level. **Significant at $1 \%$ probability level.

The 45 primers used provided uniform coverage of all three wheat genomes (A, B and D) and detected 94 polymorphic alleles (Table 6). Number of alleles ranged from one to three per SSR locus with an average of 2.1 alleles. Salt-tolerant accession 10851 amplified maximum (63) alleles followed by 4098805 (62), SARC VII and Sakha 92 (61) and 11545 and Pasban 90 (60). It was observed that salt-tolerant genotypes amplified a greater number of alleles as compared to susceptible genotypes. Twelve SSR primers (cfd 1, cfd 9, cfd 18, cfd 46, cfd 49, cfd 183, wmc 11, wmc 17, wmc 18, wmc 154, wmc 432, and wmc 503) detected specific alleles in salt-tolerant genotypes only. The level of polymorphism among the 26 genotypes was evaluated by calculating PIC values for each of the 45 SSR loci. The PIC values varied greatly for all the SSR loci tested. The number of alleles per primer ranged from one to three with an average of 2.07. Seven primers detected a single allele and their PIC value was 0 . PIC value of the remaining 38 primers ranged from 0.13 (wmc 17) to 0.66 (wmc 24, wmc 96). Pairwise estimates of similarity ranged from 0.53 (Shorawaki and 11402) to 0.95 (11186 
and 11299) and the average similarity among all 26 genotypes was 0.75 . Average genetic similarity among the salt-susceptible genotypes was $78 \%$, among the tolerant it was $76 \%$ and between the tolerant and susceptible genotypes it was $72 \%$.

\begin{tabular}{|c|c|c|c|c|c|c|}
\hline Sr. No. & Primer & $\mathrm{T}_{\mathrm{A}}$ & Size (bp) & Locus & Polymorphic allele & PIC value \\
\hline 1 & barc 12 & 52 & $150-200$ & $3 \mathrm{~A}$ & 2 & 0.56 \\
\hline 2 & barc 20 & 53 & $150-190$ & $4 \mathrm{~B} / 7 \mathrm{~B}$ & 3 & 0.38 \\
\hline 3 & barc 45 & 52 & $180-220$ & $3 \mathrm{~A} / 2 \mathrm{~B}$ & 2 & 0.74 \\
\hline 4 & barc 124 & 52 & $220-250$ & $2 \mathrm{~A} / 2 \mathrm{D}$ & 2 & 0.53 \\
\hline 5 & $\operatorname{cfd} 1$ & 60 & $150-230$ & $6 \mathrm{~A} / 6 \mathrm{~B} / 6 \mathrm{D}$ & 2 & 0.54 \\
\hline 6 & $\operatorname{cfd} 4$ & 60 & $220-270$ & 3B & 2 & 0.50 \\
\hline 7 & cfd 5 & 60 & $15-200$ & $6 \mathrm{D} / 5 \mathrm{~B}$ & 2 & 0.51 \\
\hline 8 & $\operatorname{cfd} 9$ & 60 & $150-250$ & 3D & 3 & 0.36 \\
\hline 9 & $\operatorname{cfd} 13$ & 60 & $180-254$ & $6 \mathrm{~B}$ & 2 & 0.64 \\
\hline 10 & $\operatorname{cfd} 18$ & 60 & $150-200$ & $5 \mathrm{D}$ & 2 & 0.50 \\
\hline 11 & $\operatorname{cfd} 46$ & 60 & $150-175$ & 7D & 2 & 0.79 \\
\hline 12 & $\operatorname{cfd} 49$ & 60 & $150-214$ & 6D & 2 & 0.69 \\
\hline 13 & $\operatorname{cfd} 54$ & 60 & $170-200$ & $4 \mathrm{~B} / 4 \mathrm{D}$ & 2 & 0.50 \\
\hline 14 & $\operatorname{cfd} 57$ & 60 & 291 & 5D & 1 & 0.0 \\
\hline 15 & $\operatorname{cfd} 61$ & 60 & $218-250$ & 1D & 2 & 0.61 \\
\hline 16 & cfd 66 & 60 & $202-250$ & 7D & 2 & 0.57 \\
\hline 17 & cfd 183 & 60 & $150-179$ & $5 \mathrm{D}$ & 2 & 0.82 \\
\hline 18 & wmc1 & 55 & $148-170$ & 3B & 2 & 0.65 \\
\hline 19 & wmc 3 & 55 & $250-300$ & - & 2 & 0.69 \\
\hline 20 & wme 5 & 55 & $200-250$ & - & 2 & 0.50 \\
\hline 21 & wmc 7 & 51 & 203 & 3B & 1 & 0.0 \\
\hline 22 & wmc 9 & 55 & $100-250$ & - & 3 & 0.42 \\
\hline 23 & wmc 10 & 61 & $267-300$ & $7 \mathrm{~B}$ & 2 & 0.54 \\
\hline 24 & wmc 11 & 61 & $130-250$ & $3 \mathrm{~A}$ & 3 & 0.42 \\
\hline 25 & wmc 17 & 51 & $150-200$ & $7 \mathrm{~A} / 7 \mathrm{~B}$ & 2 & 0.87 \\
\hline 26 & wmc 18 & 61 & 230 & $2 \mathrm{D}$ & 2 & 0.80 \\
\hline 27 & wmc 24 & 51 & $150-200$ & $1 \mathrm{~A}$ & 3 & 0.34 \\
\hline 28 & wmc 28 & 51 & 188 & $5 \mathrm{~B}$ & 1 & 0.0 \\
\hline 29 & wmc 96 & 61 & $250-300$ & $3 \mathrm{~A} / 4 \mathrm{~A} / 5 \mathrm{~A} / 7 \mathrm{~A} / 5 \mathrm{D}$ & 3 & 0.34 \\
\hline 30 & wmc 97 & 51 & $150-184$ & $5 \mathrm{DL}$ & 2 & 0.51 \\
\hline 31 & wmc 110 & 61 & $170-200$ & $5 \mathrm{~A}$ & 2 & 0.57 \\
\hline 32 & wmc 149 & 61 & $200-250$ & $2 \mathrm{~A} / 2 \mathrm{~B} / 5 \mathrm{~B} / 2 \mathrm{D}$ & 2 & 0.51 \\
\hline 33 & wme 154 & 61 & $147-200$ & $2 \mathrm{~B}$ & 2 & 0.68 \\
\hline 34 & wmc 258 & 61 & 293 & $4 \mathrm{~A} / 5 \mathrm{~B}$ & 1 & 0.0 \\
\hline 35 & wmc 312 & 61 & $227-260$ & $1 \mathrm{~A}$ & 2 & 0.50 \\
\hline 36 & wme 318 & 61 & 202 & $5 \mathrm{D}$ & 1 & 0.0 \\
\hline 37 & wmc 334 & 61 & 117 & 3B & 1 & 0.0 \\
\hline 38 & wmc 346 & 61 & $203-250$ & $7 \mathrm{~A}$ & 2 & 0.53 \\
\hline 39 & wmc 405 & 61 & $218-250$ & $7 \mathrm{~A} / 5 \mathrm{~B} / 1 \mathrm{D} / 7 \mathrm{D}$ & 3 & 0.36 \\
\hline 40 & wmc 406 & 61 & $217-250$ & 1B & 2 & 0.50 \\
\hline 41 & wmc 428 & 51 & 257 & 3B & 1 & 0.0 \\
\hline 42 & wmc 432 & 51 & 189 & 1D & 3 & 0.41 \\
\hline 43 & wmc 492 & 51 & $146-250$ & $3 \mathrm{~A} / 5 \mathrm{~A} / 3 \mathrm{D}$ & 2 & 0.50 \\
\hline 44 & wmc 503 & 51 & $237-300$ & 2D & 3 & 0.35 \\
\hline 45 & wmc 506 & 61 & $216-250$ & $7 \mathrm{D}$ & 3 & 0.39 \\
\hline
\end{tabular}

A dendrogram (Figure 1) was constructed based on cluster analysis of dice similarity coefficients of SSR markers. Cluster analysis grouped the 26 genotypes into four clusters. All the salt-tolerant genotypes were grouped into two clusters, A and B. All the susceptible genotypes were grouped into two clusters, $\mathrm{C}$ and D. Cluster A consisted of six salt-tolerant genotypes from Pakistan, one from Egypt and one from Iran. Cluster B consisted of eight salttolerant genotypes from Pakistan, one from Iran and one from Syria. Cluster C included two 
salt-susceptible genotypes from Italy and two from Pakistan. Cluster D consisted of two saltsusceptible genotypes from Pakistan and one from Iran. Accession 11289 was not included in any cluster (Figure 1).

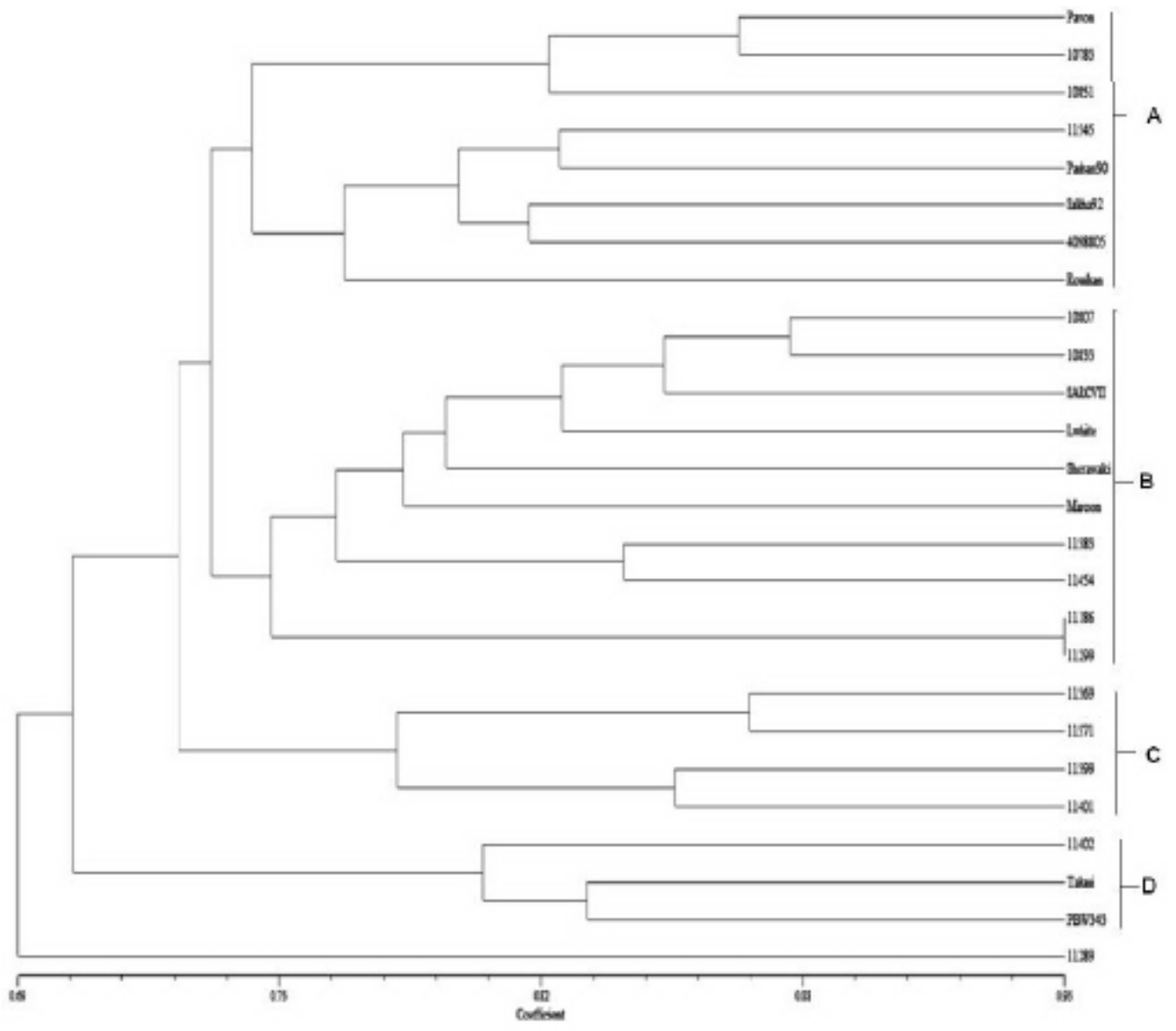

Figure 1. SSR-based genetic relationship between 26 wheat genotypes shown by UPGMA cluster analysis based on Dice similarity coefficient.

Analysis of variance of bivariate SSR data and STTI revealed that 35 SSR marker alleles significantly differentiated the salt-tolerant and -susceptible genotypes (Table 7). Shoot length, root length, number of tillers/plant, fresh shoot weight, and STI were found to be the most suitable traits to evaluate salt tolerance in wheat at vegetative stage because most of the SSR markers, which showed differences between salt-tolerant and -susceptible genotypes, showed differences with respect to these traits (Table 7). SSR markers cfd 9, cfd 18, cfd 183, wmc 96, and wmc 405 were identified as the best suited to evaluate salt tolerance because they differentiated the salt-tolerant and -susceptible genotypes on the basis of four or more traits studied (Table 7). 
Table 7. Analysis of variance for SSR data and STI under $200 \mathrm{mM} \mathrm{NaCl}$ stress.

\begin{tabular}{|c|c|c|c|c|c|c|c|c|c|c|c|c|c|c|c|c|c|c|c|}
\hline Sr. No. & Allele & SL & $\mathrm{RL}$ & TPP & FSW & FRW & DSW & DRW & STI & Sr. No. & Allele & SL & RL & TPP & FSW & FRW & DSW & DRW & STI \\
\hline 1 & cfdl & ns & ns & ns & ns & $*$ & ns & ns & $*$ & 19 & wmc10 & ns & ns & $*$ & ns & ns & ns & ns & ns \\
\hline 2 & $\operatorname{cfd} 4$ & ns & ns & ns & $*$ & ns & ns & ns & $*$ & 20 & wmc10 & ns & ns & ns & $*$ & ns & $*$ & ns & ns \\
\hline 3 & $\operatorname{cfd} 9$ & ns & ns & $*$ & ns & ns & ns & ns & ns & 21 & wmc11 & ns & ns & ns & ns & $*$ & ns & ns & ns \\
\hline 4 & $\operatorname{cfd} 9$ & $*$ & $* *$ & $*$ & $*$ & ns & $*$ & $*$ & $* *$ & 22 & wmc17 & ns & ns & ns & ns & $*$ & ns & ns & ns \\
\hline 5 & $\operatorname{cfd} 18$ & ns & ns & ns & $*$ & ns & ns & ns & ns & 23 & wmc24 & ns & ns & ns & $*$ & ns & $*$ & $\mathrm{~ns}$ & ns \\
\hline 6 & $\operatorname{cfd} 18$ & $* *$ & ns & $*$ & $*$ & ns & ns & ns & $*$ & 24 & wmc96 & $* *$ & ns & $* *$ & $* *$ & $*$ & $* *$ & $* *$ & $* *$ \\
\hline 7 & $\operatorname{cfd} 49$ & $\mathrm{~ns}$ & $*$ & ns & ns & $\mathrm{ns}$ & ns & $\mathrm{ns}$ & ns & 25 & wmc 110 & ns & ns & $\mathrm{ns}$ & ns & $\mathrm{ns}$ & $\mathrm{ns}$ & $* *$ & $\mathrm{~ns}$ \\
\hline 8 & $\operatorname{cfd} 54$ & $*$ & ns & ns & ns & ns & ns & ns & ns & 26 & wmc 149 & $* *$ & ns & ns & ns & $*$ & ns & ns & ns \\
\hline 9 & $\operatorname{cfd} 54$ & $*$ & ns & ns & ns & ns & ns & ns & ns & 27 & wmc 154 & ns & ns & $*$ & ns & ns & ns & ns & ns \\
\hline 10 & $\operatorname{cfd} 57$ & ns & $*$ & ns & ns & ns & ns & ns & ns & 28 & wmc 258 & ns & ns & $*$ & ns & ns & ns & ns & ns \\
\hline 11 & cfd66 & ns & ns & ns & ns & $* *$ & ns & ns & ns & 29 & wmc346 & ns & ns & ns & $*$ & ns & ns & ns & ns \\
\hline 12 & $\operatorname{cfd} 183$ & ns & $* *$ & $*$ & $*$ & ns & ns & $\mathrm{ns}$ & $*$ & 30 & wmc346 & ns & ns & ns & $*$ & ns & ns & $\mathrm{ns}$ & ns \\
\hline 13 & wmcl & ns & ns & $*$ & ns & $*$ & ns & ns & $*$ & 31 & wmc405 & ns & ns & ns & ns & ns & $*$ & ns & ns \\
\hline 14 & wmc1 & ns & ns & ns & ns & $*$ & ns & ns & ns & 32 & wmc405 & ns & ns & $*$ & ns & ns & ns & ns & ns \\
\hline 15 & wmc3 & ns & ns & $*$ & ns & ns & ns & ns & ns & 33 & wmc405 & $* *$ & ** & ** & * & ns & $* *$ & ns & ** \\
\hline 16 & wmc5 & $* *$ & ns & ns & $*$ & ns & ns & ns & $*$ & 34 & wmc432 & ns & ns & ns & $*$ & ns & $*$ & ns & ns \\
\hline 17 & wmc9 & ns & $* *$ & ns & ns & ns & ns & ns & $*$ & 35 & wmc503 & ns & ns & $*$ & ns & ns & ns & ns & ns \\
\hline 18 & wmc9 & ns & $* *$ & ns & ns & ns & ns & ns & ns & & & & & & & & & & \\
\hline
\end{tabular}

$\mathrm{RL}=$ root length; $\mathrm{SL}=$ shoot length, $\mathrm{TPP}=$ tillers/plant; FSW = fresh shoot weight; FRW = fresh root weight; $\mathrm{DSW}=$ dry shoot weight; DRW = dry root weight; STI = salt tolerance index. *Significant at 5\% probability level. **Significant at $1 \%$ probability level.

\section{DISCUSSION}

It is evident from the results that $\mathrm{NaCl}$ treatment had a significant inhibitory effect on all the growth attributes of wheat accessions. However, accessions differed in their response to $\mathrm{NaCl}$ stress. Salt-susceptible genotypes showed more reduction in their biomass as compared to tolerant genotypes. This was largely due to the genetic makeup of the plant, environment and genotype environment interaction. Plants are salt-sensitive at the seedling stage. It is well established that crop plants with better germination and seedling growth under salt stress will be more stress-tolerant at later stages and will produce better growth and productivity (Ahmadi and Arkedani, 2006).

Number of tillers/plant decreased with increasing $\mathrm{NaCl}$ salt stress. However, saltsensitive genotypes showed greater reduction in tillers per plant than tolerant ones. Salt stress at tiller emergence can inhibit their formation and can cause their death at later stages (Nicolas et al., 1994). This may indicate that tillers/plant and their behavior under salinity can be used to evaluate wheat genotypes in breeding programs. At $250 \mathrm{mM} \mathrm{NaCl}$ stress, most of the secondary tillers of moderately tolerant genotypes were dried, and the numbers of primary tillers of salt-sensitive genotypes were greatly reduced. Our results supported the findings of El-Hendawy (2005). High narrow sense heritability (78\%) of number of tillers/plant under 250 $\mathrm{mM} \mathrm{NaCl}$ stress and positive correlation with root fresh and dry weights and shoot fresh and dry weights made this a valuable trait as selection criteria under salt stress conditions.

Root length is the most significant trait for salinity tolerance because roots have direct contact with soil and absorb water and nutrients from soil. Therefore, root length provides important evidence of plant salinity tolerance. Greater reduction in root length under salinity stress compared to controlled condition and positive correlation at both genotypic and phenotypic levels with number of tillers/plant, shoot and root dry weights indicated that this trait was strongly associated with salinity tolerance of the plant. Therefore, root length may be poten- 
tially a good selection criterion under salt stress conditions as reported by Ashraf et al. (1986). Our results indicated that shoot growth was affected more by $\mathrm{NaCl}$ salinity than root growth. Zerihun et al. (2000) also reported that under imposed osmotic stress shoot growth inhibited more than root growth. The possible reason for the greater growth of root compared to shoot may be the fact that salt stress induces physiological drought, and plants tend to proliferate roots more at high stress levels in order to absorb more water under stress conditions. High heritability estimates (Table 3 ) and positive correlation (Tables 4 and 5) with all the growth parameters studied at both salinity levels supported the findings of Ashraf et al. (1999) that root and shoot dry weights could be used as selection criteria for salt tolerance. Dry biomass is often used as an indicator of salinity tolerance under controlled environmental conditions (Meneguzzo et al., 2000). Reduction in shoot weight of the wheat genotypes was ascribed to decreased water potential of rooting medium and growth inhibition related to osmotic effects under saline conditions (Munns et al., 1995). The ability of any genotype to maintain agronomic parameters at near control levels therefore confers salt tolerance. Dry matter production is a good criterion to evaluate the performance of plants for salt tolerance because it permits direct estimations of economic return under saline conditions (Mass, 1986). Plant traits like shoot and root fresh and dry weights are more associated with crop salt tolerance at early growth stages and can be used as selection criteria for salt tolerance (Ashraf et al., 1999).

Heritability is an important parameter for the selection of an efficient population improvement method. Single plant selection in the earlier generations may be much more effective for a character that is highly heritable as compared to one which is less heritable. Furthermore, environment may also interact with the genotypic constitution to influence heritability. Narrow sense heritability is more useful because it measures the relative importance of additive variance of genetic components that can be transmitted to offspring. High heritability estimates (above $80 \%$ ) of shoot length, tillers/plant, shoot fresh and dry weights and root fresh and dry weights under control conditions suggested that more than $80 \%$ of genetic variance transferred to offsprings was additive in nature. Therefore, selection for these traits may be proven useful during early generations. However, under stress conditions moderate heritability estimates for shoot and root length and shoot and root fresh and dry weights as compared to control conditions indicated that selection should be delayed for a few lateral generations in order to get better results.

In general, the genotypic correlation coefficients were greater than phenotypic coefficients indicating the inherent association of the traits (Tables 4 and 5). Results reported earlier by Mohammad et al. (2008) support our results. A positive association between shoot and root growth parameters indicated that for a salt-tolerant variety it is important to have healthy and long roots, which can absorb more water from deeper soils under stress condition. Healthy shoots perform photosynthesis more efficiently and contribute to root growth. A negative association between shoot length and number of tillers/plant (Tables 4 and 5) indicated that the number of tillers increases at the cost of shoot growth. Therefore, a genotype having maximum tillers and dry weight could be a better choice under salt stress conditions.

SSR markers revealed higher genetic variation in wheat genotypes, which confirmed the hydroponic evaluation. A higher level of polymorphism was observed in the landraces than in the cultivars revealed by the average allelic richness indicating that modern plant breeding has caused considerable genetic erosion in commercial wheat cultivars. Therefore, the landraces still provide a largely unexplored gene pool with great potential for broadening the genetic 
base of modern wheat cultivars. Salt-tolerant genotypes produced more alleles than sensitive ones. Generally, the salt-tolerant genotypes tend to cluster together indicating the efficiency of SSR markers to differentiate between tolerant and susceptible genotypes based on their similarity matrix. The number of alleles per primer ranged from one to three with an average of 2.07. PIC value ranged from 0.13 to 0.66 with an average of 0.40 . Coefficient of similarity matrix ranged from 0.53 to 0.95 with an average of 0.75. Almanza-Pinzón et al. (2003) reported 2 to 4 alleles per locus in 70 wheat genotypes with 37 SSR markers and a PIC value that ranged from 0.13 to 0.74 with an average of 0.45 . Bányai et al. (2006) reported an average 0.60 PIC value in 96 wheat genotypes using 15 SSR markers. Ijaz and Khan (2009) reported maximum $95 \%$ and minimum $41 \%$ similarity in 63 wheat genotypes. Our results revealed that the $D$ genome was the richest in identified SSR-based polymorphisms because $D$ genome-based SSR markers produced maximum alleles followed by $B$ and $A$ genomes (Table 6). In the present study, genotypes were selected on the basis of salt tolerance, which is largely controlled by the $D$ genome in hexaploid wheat (Gorham et al., 1987; Dvorak and Gorham, 1992). SSR markers also confirmed the phenotypic evaluation of genotypes. Twelve markers (cfd 1, cfd 9, cfd 18, cfd 46, cfd 49, cfd 183, wmc 11, wmc 17, wmc 18, wmc 154, wmc 432, and wmc 503 ) were found to be specific because they were amplified in salt-tolerant genotypes only. Nine of them located on the $D$ genome supporting the findings of Gorham et al. (1987) who reported that salinity tolerance is controlled by the $D$ genome. Analysis of variance of the SSR data and plant traits studied revealed that five SSR markers (cfd 9, cfd 18, cfd 183, wmc 96, and wmc 405) and tillers/plant, fresh shoot weight and STI were best suited to evaluate salt tolerance in wheat. SSR markers cfd 9, cfd 18 and cfd 183 also produced specific alleles in salt-tolerant genotypes only. Therefore, these primers are of great importance and may be used to screen large wheat population for salt tolerance.

Accessions were classified as tolerant, moderately tolerant, moderately sensitive, and sensitive to salt stress on the basis of the relative performance of growth parameters like shoot and root length, No. of tillers/plant, and shoot and root fresh and dry biomass production. These accessions, which acquired high STI values for the above-mentioned parameters, were salt tolerant, while those that produced less were salt sensitive. Based on STI at $200 \mathrm{mM} \mathrm{NaCl}$ stress Sakha 92 (Egypt) was the most salt tolerant followed by accessions 10793 (Pak), 10790 (Pak), 10821(Pak), and 11526 (Pak). At $250 \mathrm{mM} \mathrm{NaCl}$ stress, accession 11299 (Pak) was the most salt tolerant followed by accessions 11335 (Pak), 11370 (Italy), check cultivar Pasban 90 (Pak), 11214 (Pak), Maroon (Iran), and Sakha 92 (Egypt). Sakha 92, Pasban 90, accessions 10790, 10828, 10823, and 4098805 performed better at both 200 and $250 \mathrm{mM} \mathrm{NaCl}$ stress. High heritability estimates (above 50\%), under 200 and $250 \mathrm{mM} \mathrm{NaCl}$ stress for shoot length, shoot fresh and dry weights and root fresh and dry weights, indicate that selection may be useful in early generation. High heritability and positive correlation suggested that number of tillers/plant, root length, root fresh and dry weights and shoot fresh and dry weights are associated with salt tolerance and could be used as selection criteria under salt stress conditions. SSR markers revealed higher genetic variation in wheat genotypes, which confirmed the hydroponic evaluation. Generally, the salt-tolerant genotypes tend to cluster together indicating the efficiency of SSR markers to differentiate between tolerant and susceptible genotypes based on their similarity matrix. Twelve SSR markers were found to be specific because they were present in salt-tolerant genotypes only. These markers could be used to screen large populations for salt tolerance. The salt-tolerant genotypes will be further investigated at the 
reproductive stage and maturity in salt-affected field conditions for their confirmation as new sources of salt tolerance.

\section{ACKNOWLEDGMENTS}

The authors gratefully acknowledge the financial support from the Higher Education Commission of Pakistan and the Pakistan Agricultural Research Council. The authors also appreciate the cooperation of Drs. Abdus Salam and Javaid Akhtar at the University of Agriculture, Faisalabad, and Mr. Makhdoom Hussain at the Ayub Agriculture Research Institute, who also provided seed for these studies.

\section{REFERENCES}

Ahmadi SH and Ardekani JN (2006). The effect of water salinity on growth and physiological stages of eight Canola (Brassica napus) cultivars. Irrigation Sci. 25: 11-20.

Ali Z, Salam A, Azhar FM and Khan IA (2007). Genotypic variation in salinity tolerance among spring and winter wheat (Triticum aestivum L.) accessions. South Afr. J. Bot. 73: 70-75.

Almanza-Pinzón MI, Khairallah M, Fox PN and Warburton ML (2003). Comparison of molecular markers and coefficients of parentage for the analysis of genetic diversity among spring bread wheat accessions. Euphytica 130: 77-86.

Anderson JA, Churchill GA, Autrique JE, Tanksley SD, et al. (1993). Optimizing parental selection for genetic linkage maps. Genome 36: 181-186.

Ashraf M (2004). Some important physiological selection criteria for salt tolerance in plants. Flora 199: 361-376.

Ashraf M, McNeilly T and Bradshaw AD (1986). The response of selected salt-tolerant and normal lines of four grass species to $\mathrm{NaCl}$ in sand culture. New Phytol. 104: 453-461.

Ashraf MY, Waheed RA, Bhatti AS, Baig A, et al (1999). Salt Tolerance Potential in Different Brassica Species. Growth Studies. In: Halophyte Uses in Different Climates II (Hamdy A, Leith H, Todorovic M and Moscheuko M, eds.). Backhuys Pubs, Leiden, 119-125.

Bányai J, Szqcs P, Karsai I, Mészáros K, et al. (2006). Identification of winter wheat cultivars by simple sequence repeats (SSRs). Cereal Res. Commun. 34: 865-870.

Chatrath R, Mishra B, Ortiz Ferrara G, Singh SK, et al. (2007). Challenges to wheat production in South Asia. Euphytica 157: 447-456.

Cuartero J, Bolarín MC, Asíns MJ and Moreno V (2006). Increasing salt tolerance in the tomato. J. Exp. Bot. 57: 10451058.

Dvorak J and Gorham J (1992). Methodology of gene transfer by homoeologous recombination into Triticum turgidum: Transfer of $\mathrm{K}^{+} / \mathrm{Na}^{+}$discrimination from T. aestivum. Genome 35: 639-646.

El-Hendawy SE, Hu Y, Yakout GM, Awad AM, et al. (2005). Evaluating salt tolerance of wheat genotypes using multiple parameters. Eur. J. Agron. 22: 243-253.

El-Hendawy SE, Ruan Y, Hu Y and Schmidhalter U (2009). A comparison of screening criteria for salt tolerance in wheat under field and controlled environmental conditions. J. Agron. Crop Sci. 195: 356-367.

Gorham J, Hardy C, Wyn Jones RG, Joppa LR, et al. (1987). Chromosomal location of a K/Na discrimination character in the D genome of wheat. Theor. Appl. Genet. 74: 584-588.

Hoagland DR and Arnon DI (1950). The water-culture method for growing plants without soil. Calif. Agric. Exp. Station Circular 147: 32.

Ijaz S and Khan IA (2009). Molecular characterization of wheat germplasm using microsatellite markers. Genet. Mol. Res. 8: 809-815.

Kurup SS, Hedar YS, Al Dhaheri MA, El-Heawiety AY, et al. (2009). Morpho-physiological evaluation and RAPD markers-assisted characterization of date palm (Phoenix dactylifera L.) varieties for salinity tolerance. J. Food Agric. Environ. 7: 503-507.

Liu X, Shi J and Zhang XY (2001). Screening salt tolerance germplasm and tagging the tolerance gene(s) using microsatellite (SSR) markers in wheat. Acta Bot. Sin. 48: 948-954.

Ma L, Zhou E, Huo N, Zhou R, et al. (2007). Genetic analysis of salt tolerance in a recombinant inbred population of wheat (Triticum aestivum L.). Euphytica 153: 109-117.

Mass EV (1986). Salt tolerance of plant. Appl. Agric. Res. 1: 12-26. 
Meneguzzo S, Navari-Izzo F and Izzo R (2000). NaCl effects on water relations and accumulation of mineral nutrients in shoots, roots and cell sap of wheat seedlings. J. Plant Physiol. 156: 711-716.

Mohammad T, Amin M, Subhan F, Khan MI, et al. (2008). Identification of traits in bread wheat genotypes (Triticum aestivum L.) contributing to grain yield through correlation and path coefficient analysis. Pakistan J. Bot. 40: 2393-2402.

Munns R (2002). Comparative physiology of salt and water stress. Plant Cell Environ. 25: 239-250.

Munns R (2005). Genes and salt tolerance: bringing them together. New Phytol. 167: 645-663.

Munns R, Schachtman DP and Condon AG (1995). The significance of two-phase growth response to salinity in wheat and barley. Aust. J. Plant Physiol. 22: 561-569.

Munns R, James RA and Lauchli A (2006). Approaches to increasing the salt tolerance of wheat and other cereals. J. Exp. Bot. 57: 1025-1043.

Nicolas ME, Munns R, Samarakoon AB and Gifford RM (1994). Elevated $\mathrm{CO}_{2}$ improves the growth of wheat under salinity. Aust. J. Plant Physiol. 20: 349-360.

Pestsova E and Roder M (2002). Microsatellite analysis of wheat chromosome 2D allows the reconstruction of chromosomal inheritance in pedigrees of breeding programmes. Theor. Appl. Genet. 106: 84-91.

Prasad M, Varshney RK, Roy JK, Balyan HS, et al. (2000). The use of microsatellites for detecting DNA polymorphism, genotype identification and genetic diversity in wheat. Theor. Appl. Genet. 100: 584-592.

Rohlf FJ (2005). NTSYS pc (Numerical Taxonomy and Multivariate Analysis System). Version 2.2. Exeter Software Appl. Biostat Inc., New York.

Sabir P and Ashraf M (2007). Screening of local accessions of Panicum maliaceum L. for salt tolerance at the seedling stage using biomass production and ion accumulation as selection criteria. Pakistan J. Bot. Environ. 39: 1655-1661.

SAS Institute (2003). Release 9.1. SAS Institute Inc., Cary.

Shah ZH, Munir M, Kazi AM, Mujtaba T et al. (2009). Molecular Markers Based Identification of Diversity for Drought Tolerance in Bread Wheat Varieties and Synthetic Hexaploids. Curr. Issues Mol. Biol. 11: 101-110.

Zerihun A, Gutschick VP and Bassirirad H (2000). Compensatory roles of nitrogen uptake and photosynthetic N2 use efficiency in determining plant growth response to elevated $\mathrm{CO}_{2}$ : evaluation using a functional balance model. Ann. Bot. 86:723-730. 